\title{
Impact of Complete Dentures in the Elderly: Analysis of Bite Force and Pressure of the Tongue and Lips
}

\section{Takami Hirono Hotta ${ }^{1}$, Flávia Cristina Targa Coimbra ${ }^{1}$, Millena Mangueira Rocha $^{1}$, Marcelo Palinkas ${ }^{1,2,3 *}$, Paulo Batista de Vasconcelos ${ }^{1}$, Nicole Bar- bosa Bettiol $^{1}$, Simone Cecilio Hallak Regalo ${ }^{1,3}$, Helena de Freitas Oliveira Paranhos $^{1}$, Cláudia Helena Lovato Silva ${ }^{1}$ and Wilson Matsumoto ${ }^{1}$}

${ }^{1}$ Ribeirão Preto School of Dentistry, University of São Paulo, Brazil

${ }^{2}$ Faculty Anhanguera, Ribeirão Preto, São Paulo, Brazil

${ }^{3}$ National Institute and Technology - Translational Medicine (INCT.TM), São Paulo, Brazil

*Corresponding Author: Marcelo Palinkas, Professor, Department of Oral and Basic

Biology, School of Dentistry of Ribeirão Preto University of São Paulo, Brazil.
Received: March 09, 2020

Published: May 06, 2020

(C) All rights are reserved by Marcelo

Palinkas., et al.

\begin{abstract}
The loss of teeth can produce important alterations in the stomatognathic system, affecting bone structures, oral mucosa and occlusal force. Our study aimed to evaluate the maximum molar bite force, and pressure of the tongue and lips of elderly subjects, before and after 4 months of treatment with complete dentures. Fifteen subjects were submitted to treatment with maxillary and mandibular complete dentures. The maximum molar bite force (right and left) was measured using a digital dynamometer. The pressure of the tongue and lips were measured with the Iowa Oral Pressure Instrument (IOPI). The data were analyzed using the repeated measure test (SPSS 21.0; $\mathrm{p} \leq .05)$. Significant differences were found in the right molar bite force $(\mathrm{p}=.001)$, left molar bite force $(\mathrm{p}=$ $.001)$ and lip pressure ( $\mathrm{p}=.04)$. It was observed an increase in the maximum molar bite force and lip pressure after oral rehabilitation with complete dentures. Elderly subjects presented improved results in relation to the bite force and lip pressure after four months of treatment with complete dentures.
\end{abstract}

Keywords: Complete Denture; Elderly; Bite Force; Tongue; Lips

\section{Introduction}

With aging, it is normal to have morphological and functional changes in the stomatognathic system that may be more pronounced in the absence of the natural dentition [1,2]. In the elderly, changes due to decreased strength, tension, and resistance of the muscles and structures of the oropharynx can cause functional alterations that affect the masticatory process, deglutition and phonation [3].

The rehabilitation of the oral cavity of subjects through complete denture aims the restoration of function and aesthetic, enabling the individual to socialize without constraints caused by their oral condition [4]. However, it is common to verify that among subjects who use complete dentures, there is no concern or awareness about the guidelines regarding the use, need for reevaluations and, importantly, periodical change of prostheses. This results in their use for a prolonged period in inadequate conditions [5] that contributes to the aggravation of age-related changes.

The use of instruments that measure the functions of the stomatognathic system, such as the Iowa Oral Performance Instrument [6] and the gnathodynamometer [7], in clinical research, can facilitate the diagnosis and the prevention of alterations in the masticatory muscles, tongue, and lips by quantitative functional evaluation, which are very important for the performance analysis of the individual, especially in this age group.

\section{Objective of the Study}

The objective of this study was to evaluate the maximum molar bite force and isometric pressure exerted by the tongue and lips, before and after oral rehabilitation with complete dentures in elderly subjects. The hypothesis of this study, after oral rehabilitation with complete dentures, shows improvements in the functions of the stomatognathic system. 


\section{Materials and Methods}

Subjects

The post hoc sample size was calculated considering a level of $\alpha=0.05$, a power of $99 \%$ for the main outcome maximum molar bite force (mean [standard deviation] of right side: before, 230.75 [103.85] and after 4 months, 477.00 [207.70]) of rehabilitation with complete dentures, and effect size of 1.49. The minimal sample size obtained was 15 subjects. The sample size was calculated with the $G^{*}$ Power software v 3.0.10 (Franz Faul, Kiel University, Kiel, Germany).

A total of 15 consecutively referred subjects (with a mean (SD) age of 68.8 years (7.6) who were wearers of inadequate complete dentures and in the need for new one, participated in this study. All selected volunteers wore older upper and lower dentures.

Inclusion criteria were complete edentulism subjects with need of new complete dentures, adequate anatomical conditions for prosthetic rehabilitation and ability to perform mandibular movements of mastication (verified by opening, closing, lateral, protrusion and clenching movements) and tongue movements (such as outward, upward, downward and sideways) performed by verbal guidance of the examiner.

Exclusion criteria were subjects with presence of temporomandibular disorders (RDC/TMD), bone high, fibromucous resilience, presence of parafunctional habits (such as bruxism) and motor cognitive, and/or psychological pathologies that did not permit the execution of the research protocol, specifically, the mandibular and tongue movements.

The subjects of this study were submitted to anamnesis and clinical examination to evaluate the stomatognathic system and the complete dentures in use. The evaluation of the quality these prostheses considered some predetermined criteria indicating as adequate or not [4].

The new maxillary and mandibular complete dentures were made ensuring the adequate quality of retention, stability, aesthetic, phonetics, and maxillomandibular relationship, with bilateral balanced occlusion as occlusal scheme. After the installation, weekly reviews were scheduled to adjust the fitting and occlusal balance of the complete dentures. The subjects returned, after 4 months, for final reevaluation. The evaluation of bite force, as well as tongue and lip pressures were performed with old complete dentures and with the new ones, as described below.

\section{Ethical consideration}

This study was approved by the Research Ethics Committee with Human Beings (process No. 0205416.0.0000.5419) in compliance with Resolution CNS 466/12 which governs all clinical research in Brazil. All subjects signed the informed consent form.

Analysis of the molar bite force

The digital dynamometer (IDDK, Equipamentos Industriais Ltda, SP, Brazil) with a capacity of up to $980.665 \mathrm{~N}$ was adapted to oral conditions and used to measure maximum molar bite force $[8,9]$. The bite fork, which consists of two metal rods with plastic covers, was adequately placed between the teeth of the individual, the subjects were asked to clench the bite fork and the result readings were noted.

A trained and experienced operator made these procedures. Due to biosafety, the dynamometer rods were cleaned with alcohol and protected with disposable latex finger cots (Wariper, São Paulo, Brazil). The stems were positioned in the region of the first permanent right and left molar, where the greatest molar bite force is expected. Three readings were performed on each side, in the molar region, with an interval of two minutes between each examination and the results were mean among these three readings $[10,11]$.

\section{Maximum pressure of the tongue and lips}

The assessment of maximum tongue and lip pressures was performed using the Iowa Oral Pressure Instrument (IOPI) model 2.3 (IOPI Medical, Redmont, WA, USA), composed of a 3.5-cm-long disposable plastic bulb filled with air, which was connected to the pressure transducer by means of a $11.5-\mathrm{cm}$ plastic tube. The base with the transducer had a digital screen, where the measured values of pressure $(\mathrm{kPa})$ were visualized.

The tongue pressure was measured by the anterior elevation of the tongue with the air bulb positioned in the retro-incisal region. The subjects were instructed to raise the tongue and press the bulb against the hard palate applying maximum strength for approximately three seconds [2].

The maximal pressure exerted by the lips was measured with the bulb positioned between the lips in the midline. Subjects were instructed to keep their teeth occluded while the bulb was placed, and then to press their lips together with maximal effort [13].

Each test was performed three times, with rest intervals of 30 seconds between each time. The highest value was used as the maximum isometric pressure exerted by the subjects [14].The IOPI 
calibration was performed weekly, according to the manufacturer's instructions, to ensure the reliability of the results [12].

\section{Statistical analysis}

In the analysis of the results, the data showed normal distribution (Shapiro-Wilk normality test, $\mathrm{p} \leq .05$ ). The statistical analysis was performed statistical software IBM SPSS Statistics for Windows, version 22.0 (IBM SPSS, IBM Corp., Armonk, NY, USA). The results were obtained through descriptive analysis (average and standard deviation) for each variable. The average values were compared using the repeated measure test ( $\mathrm{p} \leq .05)$.

\section{Results}

It was observed that the average period of use of complete dentures was 29.7 years and the average time of use of the last pair of complete dentures was 15.7 years.

Table 1 shows the mean values $(\mathrm{kPa})$ for tongue and lip pressures, before and after 4 months of complete denture treatment. There was a significant difference $(\mathrm{p} \leq .05)$ in the pressure of the lips ( $p=.04)$, demonstrating greater pressure after 4 months of the oral rehabilitation.

\begin{tabular}{|l|c|c|c|}
\hline & \multicolumn{2}{|c|}{ Periods } & \multirow{2}{*}{ p value } \\
\cline { 1 - 3 } \multicolumn{1}{|c|}{ Pressure } & I & II & \\
\hline Tongue & $28.0 \pm 15.64$ & $23.66 \pm 15.61$ & .49 \\
\hline Lips & $35.40 \pm 16.64$ & $46.13 \pm 15.50$ & $.04^{*}$ \\
\hline
\end{tabular}

Table 1: Men values (kPa), standard deviation $( \pm)$, and statistical significance $\left(\mathrm{p} \leq .05^{*}\right)$ for pressure of the tongue and lips before (I) and after 4 months (II) of rehabilitation with complete dentures.

Table 2 shows the mean values $(\mathrm{N})$ of maximum molar bite force (right and left) before and after 4 months of rehabilitation with complete dentures. There was a significant difference $(\mathrm{p} \leq$ $.05)$ in the right ( $p=.001)$ and left $(p=.001)$ molar bite force after treatment, evidencing increases in the maximum molar bite force.

\begin{tabular}{|c|c|c|c|}
\hline & \multicolumn{2}{|c|}{ Periods } & \multirow{2}{*}{ P value } \\
\cline { 1 - 3 } Molar bite force & I & II & \\
\hline Right & $231 \pm 104$ & $477 \pm 208$ & $.001^{*}$ \\
\hline Left & $265 \pm 133$ & $548 \pm 252$ & $.001^{*}$ \\
\hline
\end{tabular}

Table 2: Mean, standard deviation ( \pm ), and statistical significance ( $\mathrm{p} \leq .05^{*}$ ) for right and left molar bite force ( $\mathrm{N}$ ) before (I) and after four months (II) of rehabilitation with complete dentures.

\section{Discussion}

This study aimed to make a comparative and quantitative analysis of the maxillary bite force and isometric pressure exerted by the tongue and lips, before and after four months of oral rehabilitation with complete dentures, in elderly subjects. The aging structural alterations in the musculature can promote changings in the physiological patterns $[15,16]$.

In this way, the masticatory process can become modified, and consequently no effective, with adaptations that are not always beneficial to the individual, such as choosing foods with a softer consistency [15], reduced food consumption [17,18] and muscle fatigue [19]. These are results of the difficulty in crushing the food, presence of orofacial pain and chewing discomfort.

All these alterations may be aggravated using prolonged time of complete dentures, with large wear in the artificial teeth without periodical change of them. One of the most important physical properties of artificial teeth is wear resistance to help maintaining the vertical dimension of occlusion and masticatory efficiency, however, due to peculiar characteristics of each individual as mastication, swallowing, occurrence or not of parafunctional occlusal contacts and time of use, the teeth and other components of the prosthesis could be worn [20]. As there is a relationship between the quality of the prosthesis and masticatory performance [18], under the mentioned conditions, the action of muscular forces may displace complete dentures during mastication and leading to impairment function.

With the loss of vertical dimension of occlusion, the functions of speech, chewing, and swallowing may be distorted or performed through adaptations that may compromise the stomatognathic system, such as, interposition of the tongue and compensatory use of muscles which are not directly related to those functions [1].

With aging, there is a tendency for reduced tongue and lip strength [15] and this could be associated to longer feeding time, reduced food consumption and difficulties in swallowing that contribute the masticatory process be a very arduous and tiring task [17].

Additionally, considering that a good proportion of the elderly use complete denture, when it presents with structural and biomechanical constraints such as poor retention and lack of stability, they may demand excessive work to be performed by the tongue, lips and cheek muscles in an attempt to functionally adapt the stomatognathic system to the existing dental conditions. 
In addition to the difficulties of old age, there are also those due to inadequate prosthetic devices. In this study, the average age of the subjects was 68.8 years, the average period of use of the prostheses was 29.7 years, and the average time of the use of last complete dentures was 15.7 years, that is, a sample of elderly subjects with the history of wearing the same prostheses for a long time, probably, with structural alterations.

Complete dentures should be adjusted over time to compensate the progressive tissue changes because subjects who use conventional prostheses often complain of instability, which leads to inadequate chewing and insecurity [18]. It is essential that the individual be evaluated not only based on the adequacy of the prosthetic apparatus, but also, the functional suitability of the same.

Thus, in addition to the adequate instructions regarding the periodic replacement of the prostheses, this evaluation may suggest complementary therapies such as phonoaudiology and physiotherapy [21], aiming at the general welfare of the individual, through prevention and additional care.

Strategies for strengthening the tongue and lips can be developed and refined to deal with disabilities such as weakness of the soft tissues [15] and masticatory muscles, even if this weakness be attributed to the advanced age. These can aid in controlling the mobility of the mandible and the tongue while grinding food and ensure the correct transport of the food bolus [22] without diverting its route to the upper airways, which may cause disorders to the individual.

The use of IOPI can be a way of analyzing of the tongue and lips force. Some authors have suggested that healthy elderly should have maximum isometric pressure of at least $40 \mathrm{kPa}$ [16]. However, Machida., et al. [22] reported pressure around $26.3 \pm 7.8 \mathrm{kPa}$ for men and $24.6 \pm 7.2 \mathrm{kPa}$ for women, with no significant difference between genders. In this study, initial mean values were 37 $\mathrm{kPa}$ and after rehabilitation $42 \mathrm{kPa}$, values close to those found by Namasivayam., et al. [17] in healthy subjects, this suggests that oral rehabilitation was successful in this regard.

The assessment of bilateral maximum molar bite force in subjects who use complete dentures is an important analysis because it is involved in several critical stages of food breakage [9]. Bite force and masticatory muscle activity are reduced in subjects with complete denture, as compared to dentate subjects [23]. Tooth loss and consequent prosthetic replacement decrease between 20 and $50 \%$ of bite strength in relation to natural dentition [24]. It also reduces masticatory performance to one-fourth to one-seventh of the performance of dentate subjects, depending on age and type of food [18].

In the present study, before oral rehabilitation, the bite force was $23.5 \pm 10.2 \mathrm{~N}$ (right molar region) and $27 \pm 13.5 \mathrm{~N}$ (left molar region). With the new complete dentures after four months of use, the values changed to $48.6 \pm 21.2 \mathrm{~N}$ (right molar region) and $55.9 \pm 25.9 \mathrm{~N}$ (left molar region), with a statistically significant difference between the values obtained before and after 4 months of oral rehabilitation. This suggests better activity of the masticatory muscles with new complete dentures. These values are below those found by Sharma., et al. [18] and above those found by Hotta., et al. [10], therefore, this variation can be attributed to age, gender, adjustments of the prostheses and time of adaptation to the new prostheses. These discrepancies warrant the need for further studies.

The present study had some limitations with the salivary flow rate of all subjects, because it can influence the adaptation and use of dentures. In addition, drugs used by subjetcts should also be considered, especially those that can decrease salivary flow. The hypothesis of this study was confirmed, complete dentures improved the maximal molar bite force and tongue and lip force. Finally researches that analyzes the functional alterations in elderly subjects rehabilitated with complete dentures should have as objectives the diagnosis, prevention and indicate options that can eliminate or reduce deleterious effects on structures of the stomatognathic to promote a better quality of life for this population.

\section{Conclusion}

Based in the results of this study, elderly subjects presented better results in relation to the bite force and lip pressure after four months of the treatment with complete dentures. The results found in this study can be very important in the awareness of the dentist and the patient about the necessity of periodical changes of the complete dentures.

\section{Acknowledgement}

This study was supported by the São Paulo Research Funtation (FAPESP 2015/09942-4) and National Institute and Technology Translational Medicine (INCT.TM). The authors have no professional relationships with companies or manufacturers who will benefit from the results of the present study.

\section{Bibliography}

1. Abrams AP., et al. "Physiology of aging of older adults - Systemic and oral health considerations". Dental Clinics of North America 58 (2014): 729-738. 
2. Baumgarten A., et al. "Dental status, oral prosthesis and chewing ability in an adult and elderly population in southern Brazil". Clinics (Sao Paulo) 72 (2017): 681-685.

3. Schimmel M., et al. "Masticatory performance and maximum bite and lip force depend on the type of prosthesis". The International Journal of Prosthodontics 30 (2017): 565-572.

4. Palla ES., et al. "Soft denture liners' effect on the masticatory function in patients wearing complete dentures: A systematic review". Journal of Dentistry 43 (2015) :1403-1410.

5. Chen JH., et al. "Dentist-patient communication and denture quality associated with complete denture satisfaction among Taiwanese elderly wearers". The International Journal of Prosthodontics 28 (2015): 531-537.

6. Azevedo ND., et al. "Tongue pressure measurement in children with mouth-breathing behaviour". Journal of Oral Rehabilitation 45 (2018): 612-617.

7. Gonçalves LMN., et al. "Alterations in the stomatognathic system due to amyotrophic lateral sclerosis". Journal of Applied Oral Science 26 (2018): e20170408.

8. Palinkas M., et al. "Age and gender influence on maximal bite force and masticatory muscles thickness". Archives of Oral Biology 55 (2010): 797-802.

9. Palinkas M., et al. "Alterations in masticatory cycle efficiency and bite force in individuals with periodontitis". International Journal of Health Sciences (Qassim) 13 (2019): 25-29.

10. Hotta PTH., et al. "Bite force in temporomandibular dysfunction (TMD) and healthy complete denture wearers". Brazilian Dental Journal 19 (2008): 354-357.

11. Bonjardim LR., et al. "Association between symptoms of temporomandibular disorders and gender, morphological occlusion, and psychological factors in a group of university students". Indian Journal of Dental Research 20 (2009): 190-194.

12. Potter NL, et al. "Maximal tongue strength in typically developing children and adolescents". Dysphagia 24 (2009): 391-397.

13. Clark HM., et al. "Age and sex differences in orofacial strength". Dysphagia 27 (2012): 2-9.

14. Vanderwegen J., et al. "The influence of age, sex, bulb position, visual feedback, and the order of testing on maximum anterior and posterior tongue strength and endurance in healthy Belgian adults". Dysphagia 28 (2013): 159-166.
15. Stierwalt JAG., et al. "Tongue measures in individual with normal and impaired swallowing". American Journal of SpeechLanguage Pathology 16 (2007): 148-156.

16. Fei T., et al. "Age-related differences in tongue-palate pressures for strength and swallowing tasks". Dysphagia 28 (2013): 575581.

17. Namasivayam AM., et al. "The effect of tongue strength on meal consumption in long term care". Clinical Nutrition 35 (2016): 1078e-1083e.

18. Poluri A., et al. "Fatigue in the elderly population". Physical Medicine and Rehabilitation Clinics of North America 16 (2005): 91-108.

19. Munshi N., et al. "In vitro wear resistance of nano-hybrid composite denture teeth". Journal of Prosthodontics 26 (2017): 224-229.

20. Sharma AJ., et al. "A comparative evaluation of chewing efficiency, masticatory bite force, and patient satisfaction between conventional denture and implant-supported mandibular overdenture: An In vivo study". The Journal of Indian Prosthodontic Society 17 (2017): 361-372.

21. Sugiyama T., et al. "Effect of swallowing exercises in independent elderly". The Bulletin of Tokyo Dental College 54 (2013): 109-115.

22. Machida N., et al. "Effects of aging and sarcopenia on tongue pressure and jaw-opening force". Geriatrics and Gerontology International 17( 2017): 295-301.

23. Al-Jabrah OA., et al. "Prevalence of temporomandibular disorder signs in patients with complete versus partial dentures". Clinical Oral Investigations 10 (2006): 167-173.

24. Shala K., et al. "Evaluation of maximum bite force in patients with complete dentures". Open Access Macedonian Journal of Medical Sciences 6 (2018): 559-563.

\section{Assets from publication with us}

- Prompt Acknowledgement after receiving the article

- Thorough Double blinded peer review

- Rapid Publication

- Issue of Publication Certificate

- High visibility of your Published work

Website: www.actascientific.com/

Submit Article: www.actascientific.com/submission.php

Email us: editor@actascientific.com

Contact us: +919182824667 